\title{
HANDHELD FISHEYE MULTICAMERA SYSTEM: SURVEYING MEANDERING ARCHITECTONIC SPACES IN OPEN-LOOP MODE - ACCURACY ASSESSMENT
}

\author{
L. Perfetti ${ }^{1}$, F. Fassi ${ }^{1}$ \\ ${ }^{1}$ Politecnico di Milano, Dept. of Architecture, Built environment and Construction engineering (ABC), Italy \\ (luca.perfetti, francesco.fassi)@polimi.it)
}

\section{Commission II}

KEY WORDS: Multicamera, Fisheye, Photogrammetry, Mobile mapping, Architecture, Cultural Heritage

\begin{abstract}
:
The task of digitalizing meandering complex spaces in 3D is a challenging one even with the most advanced instrumentation like lightweight terrestrial laser scanner or portable/wearable Mobile Mapping Systems (MMSs). The complexity and extension of architectonic spaces such as staircases, corridors and passages are such that the acquisition time using static devices becomes prohibitive and the accuracy using mobile devices gets affected by drift error leading to warped models or requiring abundant control measurements. This paper presents a photogrammetric portable fisheye multicamera solution for the 3D survey of complex areas that aims at being both handy and fast in the acquisition as well as more reliable ad accurate than common MMSs. The paper showcases a stress test conducted on five complex reconstruction trajectories selected from the meandering connection passages of Milan's Cathedral. The tests are constructed as worst-case scenario to evaluate the accuracy and drift error amount of the proposed system in open-ended unconstrained paths. The results, though still suffering from moderate drift error, highlights the potential of the solution, especially in retaining the overall shape and orthogonality of the architectonic elements acquired.
\end{abstract}

\section{INTRODUCTION}

The process of three-dimensional digitization of the built environment, especially of the valuable cultural heritage architectures, is today of imperative importance, required by management, administration, and technical offices. This process is essential so that many other activities can follow, such as: maintenance, management, conservation, and cultural valorisation of these assets. To this end, there are now many established practices and continuous innovation aimed at surveying the built environment in 3D. The different techniques and instruments used, such as terrestrial photogrammetry, terrestrial laser scanners and portable laser scanners, offer as final output of the survey a $3 \mathrm{D}$ point cloud able to describe the geometry of the surveyed spaces by itself or, able to serve as a basis for the subsequent phase of restitution or modelling. Regardless, therefore, of the tools used in the survey phase, the final output is comparable between the different approaches and therefore the choice to use one or another instrumentation depends primarily on technical evaluations regarding the feasibility of operations: speed, cost, practicality, and the accuracy and resolution required to the final data.

One of the main critical elements in the digitization of architectural heritage is the problem of mapping those areas characterized by poor accessibility: such as tunnels, corridors, stairwells, service spaces, and narrow spaces in general, for which the instrumental options to choose from are reduced.

Measuring instruments designed to be handy, portable, and fast in data acquisition are increasingly present on the market today. Among these, in the category of terrestrial laser scanners there is the Leica RTC360; while in the category of portable scanners we find handheld solutions such as Geoslam ZEB REVO, Kaarta Stencil and Contour; backpack solutions such as Leica Pegasus Backpack and Gexcel Heron; and even drone solutions for indoor use, such as those produced by Emesent. However, all these solutions do not yet satisfy all scenarios required for the digitization of built heritage. Among the main limitations are: (i) the poor accuracy of mobile mapping systems when they have to be used in long acquisitions in open-loop mode; (ii) the minimum scanning range of the sensors used by both terrestrial and portable scanners when they have to be used in confined spaces; and (iii) the complexity of instrument handling and consequent lengthening of acquisition times in the field with regard to even the most manageable terrestrial scanners when they have to be used in complex confined spaces.

On the other hand, there are no commercial solutions on the market that exploit a photogrammetric approach, which has great potential to overcome the current limitations of the state of the art.

This paper presents a new measuring instrument (Figure 1, patent pending No. 102021000000812): a fisheye multicamera device that can be used on the move and operated handheld by a single operator, designed and prototyped for the rapid threedimensional survey of complex and narrow architectural spaces. The purpose of this instrument is to obtain a complete and accurate survey, in the form of a point cloud, in a short time that can facilitate the acquisition of complex narrow spaces and thus promote complete three-dimensional digitization in all its parts even of the most complex architecture.

The objective of this paper is to evaluate the accuracy and reliability of this multicamera system through field tests, in particular the robustness of the system with respect to error propagation in long acquisitions in open-loop mode.

\subsection{Related Works}

Multi-camera photogrammetric systems designed for mobile mapping are widespread in the literature where these are chosen mainly for three reasons: (i) the speed of acquisition in the field, (ii) the cost-effectiveness of the solution in comparison with the state of the art, (iii) the possibility of automatically scaling the three-dimensional reconstruction based on the known dimensional relationships between the rigid cameras composing the system. Among the authors that proposed a multicamera system for the acquisition of extensive narrow spaces there are: Koehl et al., (2016), who evaluated different stereo configurations of four GoPro action cameras mounted on a rigid bar for the fast 3D reconstruction of urban tunnels; Meyer et al. (2020), that presented a system composed of multiple off-theshelf mirrorless cameras to achieve the fast $3 \mathrm{D}$ reconstruction of tunnels and human-equivalent inspection; Panella et al., (2020), 
that presented an evaluation comparison test between a multicamera array of GoPro cameras and a terrestrial laser scanner. Other authors presented successful application of stereo multicameras to be employed underwater (Shortis et al., 2007; Nocerino et al., 2018; Nocerino et al., 2019) exploiting the advantages in scaling the results without the need of additional measurements. While others presented and tested multi-camera panoramic configurations: Teo (2015) used a multi-GoPro panoramic rig to survey indoor environments and staircases, Barazzetti et al. (2017) presented some results using the commercial Samsung Gear360 pano-camera, while Barazzetti et al. (2020) proposed a novel approach to integrate $360^{\circ}$ imagery in restoration projects.

Our approach involves a rig in which the cameras are arranged in a stereoscopic configuration and exploits the use of fisheye lenses and their wide angle of view to survey narrow spaces (Perfetti et. al., 2017).

\section{MULTICAMERA SYSTEM}

The proposed multi-camera system (Figure 1) is a portable device that consists of a hand-held probe containing the mapping unit and a small backpack housing the power supply and a computer. The mapping unit consists of five industrial grade cameras (Flir BFS 50S5) employing a 2/3" colour sensor with a resolution of $2448 \times 2048$ pixels and a detector pitch of $3.45 \mu \mathrm{m}$ and of LED lamps that points to the front and to the sides of the device for indoor use. The cameras are coupled with fisheye lenses that span a field of view of $190^{\circ}$ allowing overall imaging of the entire surroundings of the instrument except for the operator. From the probe, the operator can control the automatic acquisition of a series of images while autonomously navigating the environment to be measured.

The cameras in the system are arranged according to Perfetti (2020) aimed at being most effective when used in narrow tunnel (around $1 \mathrm{~m}$ wide) with significant separation between one another (distance between side front cameras and side back cameras are $\sim 220 \mathrm{~mm}$ ).

Of fundamental importance for the system is the stability of the rig geometry during the movement, this is ensured by the synchronization of the camera captures and by the use of global shutter sensors.

An adequate synchronisation of the cameras ensures that the location of the cameras at the moment of image capture reflects the geometry of the multicamera at rest even while moving, thus avoiding delays between acquisitions to distort the relative location of the images. The synchronisation of the system has been tested for accuracy as in Perfetti (2020) and resulted in a max synch error of $\sim 200 \mu$ s which fits the intended use case scenario of the device that is indented to be used at walking speed.

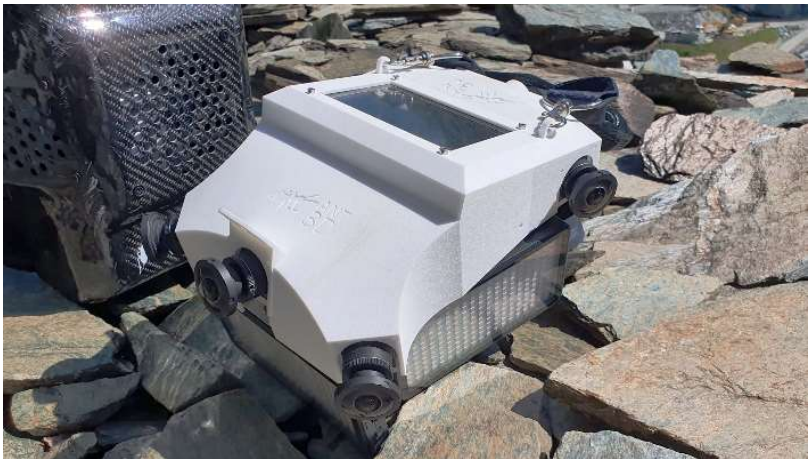

Figure 1. Picture of the prototype of the multicamera system.
The use of global shutter sensors is also important for the cameras to be used in motion avoiding the distortion introduced by the rolling shutter effect so that accurate multicamera constraints can be exploited during processing.

The main advantage of the proposed multicamera is its manoeuvrability combined with the ease of use that allows to acquire in a single sequence complex environments and different areas such as outdoor environments, indoor environments and detailed elements like niches. Moreover, provided that the cameras' shooting speed is set correctly, the acquisition does not suffer from the shaking or vibrations caused by uncertain terrain or complex or chaotic acquisitions.

\section{ACCURACY ACCESSMENT}

With the aim to evaluate the performance of the proposed multicamera system, it was decided to test it in challenging tasks and to simulate worst case scenarios. A case study was identified that had the necessary characteristics in terms of complexity and accessibility of spaces to test the proposed approach highlighting its limitations. The chosen case study consists of five narrow paths that are part of the service passages of the Milan Cathedral. These paths (Figure 2 and Figure 3) were chosen according to their length and complexity. These paths wind along the spaces of the cathedral, intercepting environments with different characteristics such as: spiral staircases, internal passages, small rooms, external areas, illuminated environments and completely dark environments. Aside for "Path A", the paths are open-ended: the acquisition with the multicamera starts at one end of the path and end on the opposite end. A return acquisition is also usually performed, but the return acquisition follows the same trajectory of the outward acquisition. The whole winding of the paths is left unconstrained and check points ( $\mathrm{CPs})$ are used to measure the drift error

For all tests the accuracy of the reconstruction was assessed with two checks: (i) a "global check", where all points available are used as CPs; and (ii) a "max drift check", where only the beginning of one end of the open loop is constrained and the drift error is measured on all other CPs.

For the "max drift check" the constraint points are used as ground control points (GCPs) in a seven parameters similarity transformation and the opposite end is used as check. This is done with the intention of simulating the worst-case scenario of an open-ended extensive path, for which no GCPs are available, to experimentally estimate the drift error of the proposed system.

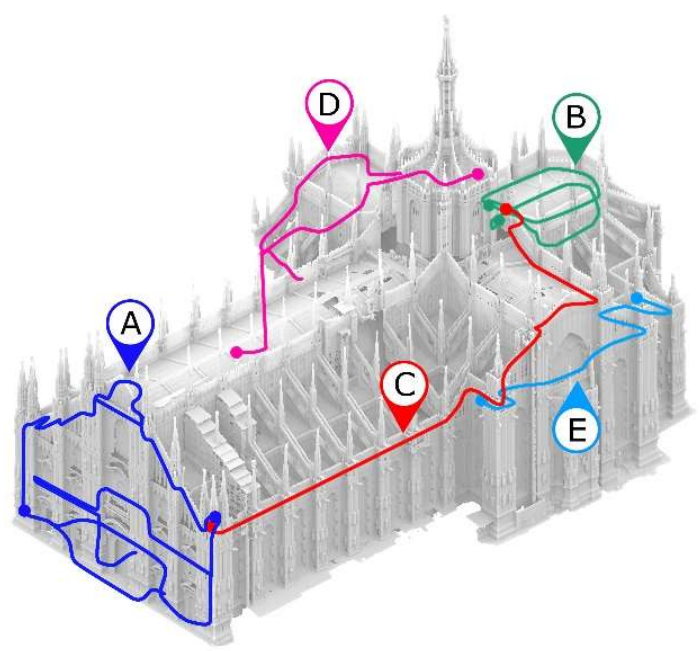

Figure 2. test paths acquired with the multi-camera on an orthographic view of the Milan's Cathedral. 

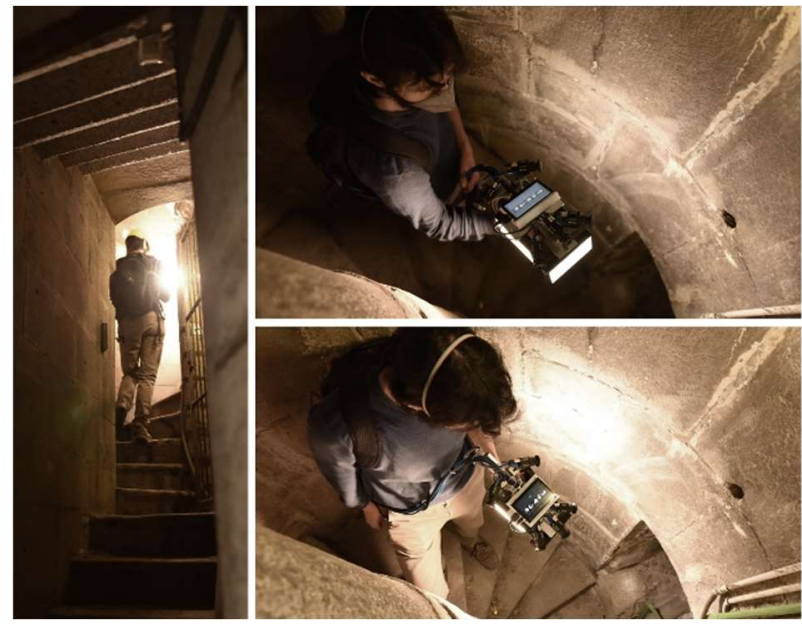

Figure 3. On field acquisitions inside a narrow spiral staircase.

\subsection{The test paths}

3.1.1 Path A: this path is the only closed loop dataset; it covers all passages hidden in the Milan Cathedral façade including 3 narrow spiral staircases $\sim 70 \mathrm{~cm}$ wide (Figure 6 ). The acquisition took $70 \mathrm{~min}$ to complete for a length of $290 \mathrm{~m}$.

3.1.2 Path B: this path starts on the rooftop of the apse and connects to a series of rooms at the extrados of the apse vaults (Figure 7). The acquisition was $295 \mathrm{~m}$ long and took $35 \mathrm{~min}$.

3.1.3 Path C: this passageway connects the high level of the cathedral roofs with the lower level and continues until it reaches the façade (Figures 7). This acquisition was performed in the forward direction only for a length of $245 \mathrm{~m}$ completed in $15 \mathrm{~min}$. 3.1.4 Path D: this passage focuses on the north transept, connecting the church level with the rooftop level via a long spiral staircase (Figures 8). The whole acquisition took $120 \mathrm{~min}$ and covered a length of $465 \mathrm{~m}$.

3.1.5 Path E: this passage is concentrated at the low level of the north transept roofs (Figures 9), is $256 \mathrm{~m}$ long and took 45 min to be acquired.
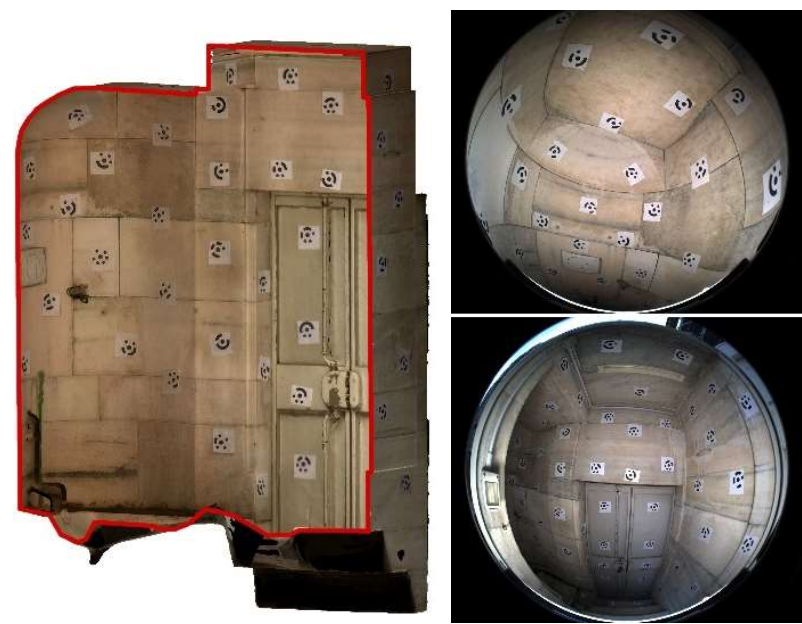

Figure 4. Section view of the calibration testfiled used (left), and two images acquired by the multi-camera system (right).

\section{CALIBRATION}

The multicamera system was carefully calibrated to take advantage of the rig rigid configuration in improving the reconstruction accuracy. This was done by calculating the internal orientation parameters of the individual cameras and the relative orientation parameters between them:

4.1.1 Internal orientation parameters: they were calculated using a strongly textured test-field of known coordinates (Perfetti et. al., 2018; Perfetti, 2020). The calculated parameters were then used as initial values in the following data processing performed using Agisoft Metashape.

4.1.2 Multicamera relative constraint: regarding the relative orientations, only the baselines between the cameras were calculated and not the relative rotations. These were then used as constraints in the processing in Metashape.

\begin{tabular}{|l|l|l|l|l|l|l|l|l|l|l|l|}
\cline { 3 - 11 } \multicolumn{2}{c|}{} & $1-2$ & $1-3$ & $1-4$ & $1-5$ & $2-3$ & $2-4$ & $2-5$ & $3-4$ & $3-5$ & $4-5$ \\
\hline \multirow{4}{*}{ C1 } & median & 258.44 & 101.18 & 94.42 & 254.86 & 290.29 & 221.82 & 203.17 & 173.99 & 214.04 & 285.35 \\
& MAD & 0.17 & 0.15 & 0.11 & 0.11 & 0.16 & 0.10 & 0.24 & 0.13 & 0.08 & 0.18 \\
& median (FD) & 258.41 & 101.15 & 94.42 & 254.83 & 290.27 & 221.80 & 203.14 & 173.98 & 214.03 & 285.32 \\
\hline \multirow{3}{*}{ C2 } & median & 258.45 & 101.19 & 94.43 & 254.86 & 290.31 & 221.83 & 203.17 & 174.00 & 214.03 & 285.34 \\
\cline { 2 - 12 } & MAD & 0.16 & 0.15 & 0.11 & 0.13 & 0.17 & 0.11 & 0.24 & 0.11 & 0.08 & 0.19 \\
\cline { 2 - 11 } & median (FD) & 258.41 & 101.17 & 94.43 & 254.83 & 290.28 & 221.81 & 203.16 & 173.98 & 214.02 & 285.30 \\
\hline
\end{tabular}

Table 1. Estimated baselines obtained from calibration 1 before the acquisition $(\mathrm{C} 1)$ and calibration 2 after the acquisition (C2). The table reports the median and MAD of the whole dataset and the median of the filtered dataset (FD) after outlier removal. All values are expressed in millimetres.

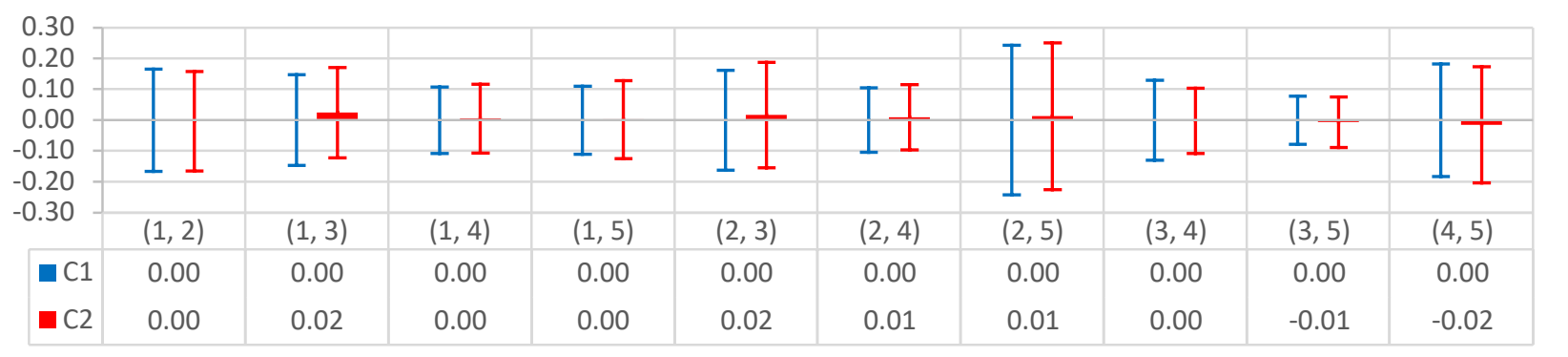

Figure 5. Difference between the baselines computed in calibration $1(\mathrm{C} 1)$ and in calibration $2(\mathrm{C} 2) \pm \mathrm{MAD}$. The comparison is based on the values obtained from the filtered dataset. Values expressed in millimetres. 


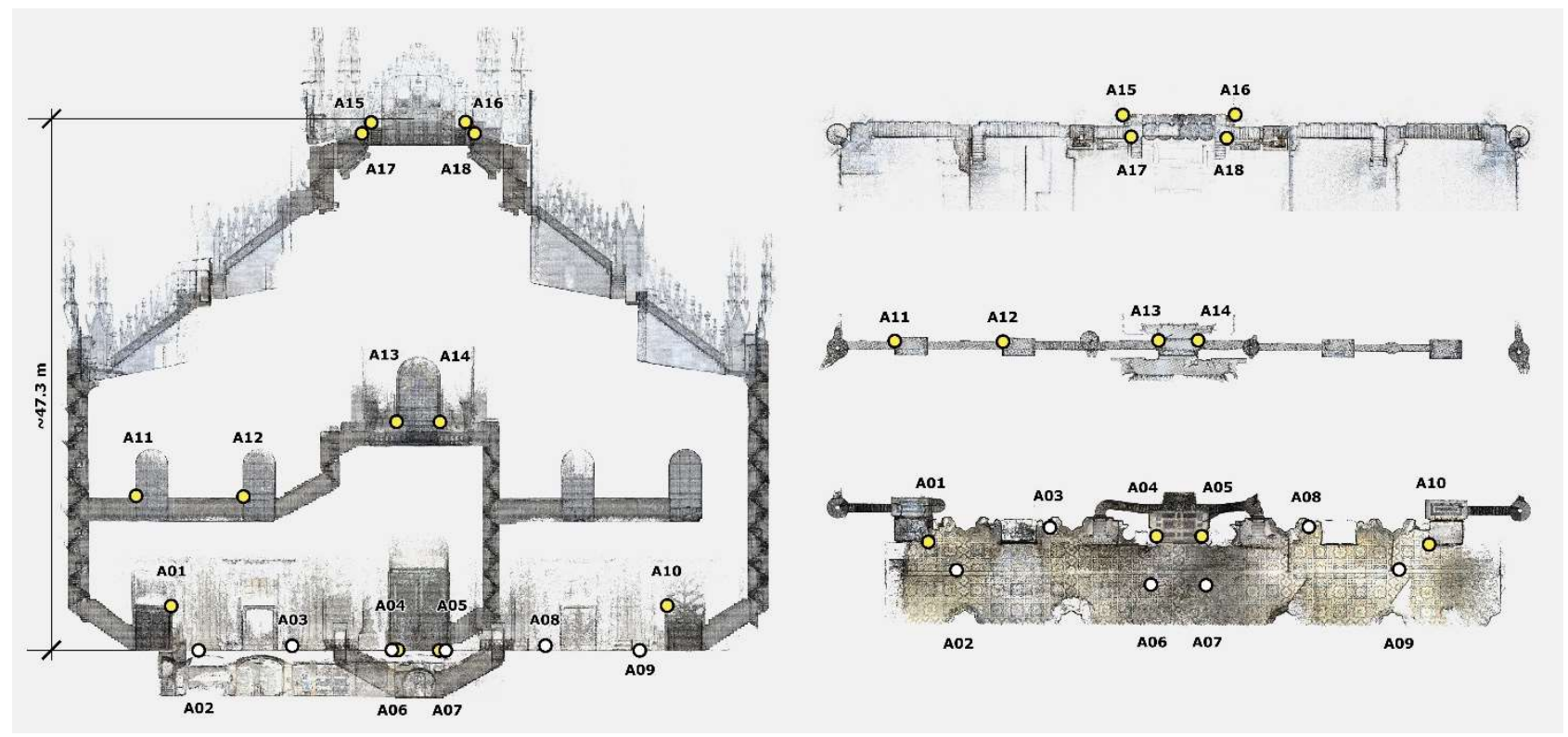

Figure 6. Point cloud result of Path A. Elevation view (left) and top view at three levels (right). According to Table 2, points in white are used as GCPs in the "max drift check".

A check on the stability of the multicamera system was also performed by repeating the baseline computation twice: once before performing acquisitions for the test, and a second one at the end of the tests. The results obtained are described below.

To perform the calibration, a small room, available on site, was set up for the task (Figure 4). The room measures approximately $\mathrm{L}: 2 \mathrm{~m} ; \mathrm{W}: 1 \mathrm{~m} ; \mathrm{H}: 2$, it has a good texture to be used for the Structure from Motion (SfM) and was also set up with photogrammetric markers whose coordinates were accurately derived. Using the calibration room, two image datasets were acquired with the multicamera system by programming a timed acquisition at 1 frame per second and rotating the system in all directions. The two datasets were then processed to derive for each camera pair a sample of approximately 100 estimated distances.

The values of the baselines were then computed by taking the median of these samples as a robust estimator was needed in the presence of outliers. Table 1 shows the values obtained for each camera pair for both calibrations $(\mathrm{C} 1$ and $\mathrm{C} 2)$ along with the Median Absolute Distance from the median (MAD) as an indicator of sample dispersion as Nocerino et. al. (2018). Optimized baselines values were finally calculated by computing the median of the filtered datasets (FD) of the samples, after removing outliers (Table 1). Outliers were identified using the modified Z-score (1) as presented by Iglewicz \& Hoaglin (1993), a threshold of $M_{i} \geq 3.0$ was chosen to identify and discard outliers.

$$
M_{i}=\frac{0.6745\left(x_{i}-\tilde{x}\right)}{M A D}
$$

where $\tilde{x}=$ median

$M A D=$ median of the absolute distances from the median

Finally, a comparison was made between the two calibrations obtained $\sim 2$ hours apart, before and after the tests were carried out. The graph and the table in Figure 5 show the difference between the distances obtained from the two calibrations $(\mathrm{C} 1$ and $\mathrm{C} 2$ ) and the values calculated by $\mathrm{C} 1$. It is possible to see how the difference between the two calibrations are negligible: max $0.02 \mathrm{~mm}$, far below the uncertainty of the calibration.

\section{RESULTS}

The acquired dataset for each of the five paths (Figure 2) were processed using Agisoft Metashape to carry out the SfM and to perform the evaluations. The computed baselines were imposed as constraints during the image orientation together with a preliminary calibration of the interior orientation parameters. At the end of the process the reconstructions were checked for gross errors that would eventually be fixed manually by re-orienting incorrectly positioned images. For the most part the acquisitions did not require manual processing after SfM.

\begin{tabular}{|c|c|c|c|c|c|c|c|c|}
\hline \multirow[b]{3}{*}{ ID } & \multicolumn{8}{|c|}{ Path A } \\
\hline & \multicolumn{4}{|c|}{ global check } & \multicolumn{4}{|c|}{ max drift check } \\
\hline & Err. & $\mathrm{x}$ & $\mathrm{y}$ & $\mathrm{z}$ & Err. & $\mathrm{x}$ & $\mathrm{y}$ & $\mathrm{z}$ \\
\hline A01 & 3.7 & -1.4 & 2.2 & -2.6 & 3.5 & -0.8 & 3.1 & 1.5 \\
\hline $\mathrm{A} 02$ & 5.0 & -2.4 & 1.9 & -3.9 & 3.4 & -2.4 & 2.4 & 0.4 \\
\hline $\mathrm{A} 03$ & 3.2 & -0.9 & 1.1 & -2.9 & 1.8 & -0.9 & 1.4 & 0.6 \\
\hline A04 & 3.6 & -0.6 & -0.3 & -3.5 & 0.8 & -0.8 & -0.2 & -0.2 \\
\hline A05 & 3.8 & -0.9 & -0.5 & -3.7 & 1.3 & -1.2 & -0.4 & -0.5 \\
\hline A06 & 4.6 & -1.6 & -0.4 & -4.3 & 1.9 & -1.9 & -0.2 & -0.3 \\
\hline A07 & 5.2 & 0.3 & -1.4 & -5.0 & 1.8 & 0.0 & -1.3 & -1.2 \\
\hline A08 & 5.0 & 3.5 & 0.2 & -3.5 & 3.3 & 3.2 & 0.1 & -0.8 \\
\hline A09 & 3.8 & 2.6 & -2.2 & -1.6 & 3.5 & 2.0 & -2.4 & 1.4 \\
\hline $\mathrm{A} 10$ & 4.0 & 3.0 & -2.2 & -1.5 & 3.8 & 3.0 & -2.1 & 1.1 \\
\hline A11 & 2.2 & -1.0 & 1.8 & 0.7 & 5.7 & 0.8 & 3.6 & 4.3 \\
\hline $\mathrm{A} 12$ & 1.0 & 0.4 & 0.7 & 0.6 & 5.0 & 2.2 & 2.3 & 3.9 \\
\hline A13 & 5.4 & -3.4 & -2.0 & 3.7 & 6.5 & -1.0 & 0.0 & 6.4 \\
\hline A14 & 5.6 & -2.6 & -3.0 & 4.0 & 6.7 & -0.2 & -1.1 & 6.7 \\
\hline A15 & 6.1 & 0.3 & 0.6 & 6.0 & 11.7 & 5.9 & 5.1 & 8.8 \\
\hline A16 & 6.7 & 3.2 & 1.7 & 5.7 & 13.3 & 8.7 & 6.0 & 8.1 \\
\hline A17 & 5.7 & 0.6 & 1.0 & 5.6 & 12.0 & 6.4 & 5.5 & 8.6 \\
\hline A18 & 6.0 & 1.3 & 0.3 & 5.8 & 12.0 & 6.9 & 4.7 & 8.5 \\
\hline $\max$ & 6.7 & 3.2 & 1.7 & 5.7 & 13.3 & 8.7 & 6.0 & 8.1 \\
\hline
\end{tabular}

Table 2. Error check of Path A. For "global check" all points are used as CPs, while for "max drift check" points highlighted in grey (white in Figure 6) are used as GCPs in a similarity transformation. All errors are expressed in centimetres. 


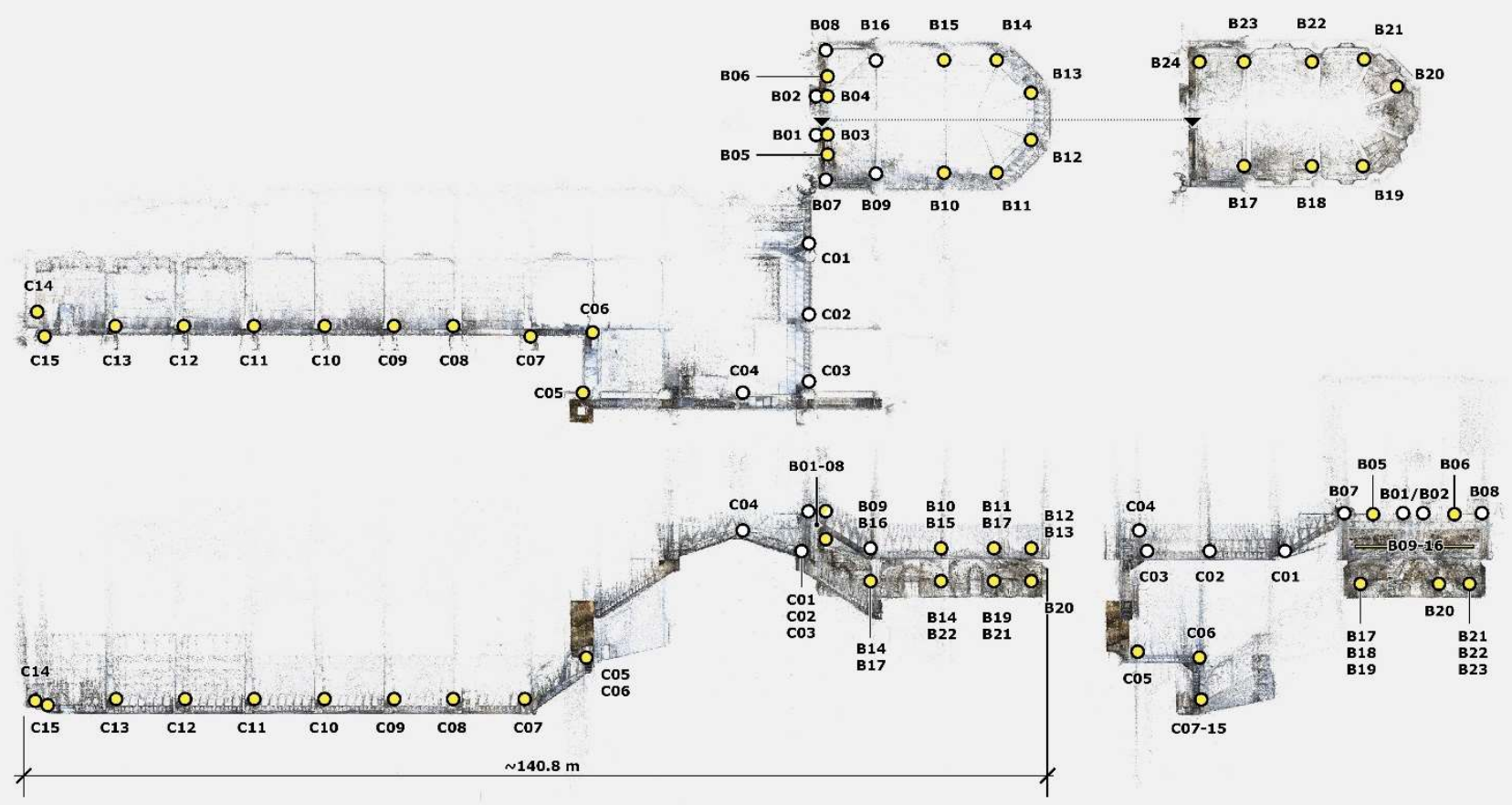

Figure 7. Point cloud result of Path B and Path C. Top views (top) and elevation views (bottom): front elevation bottom left and side elevation bottom right. According to Table 3 "Path B" and "Path C", points in white are used as GCPs in the "max drift check".

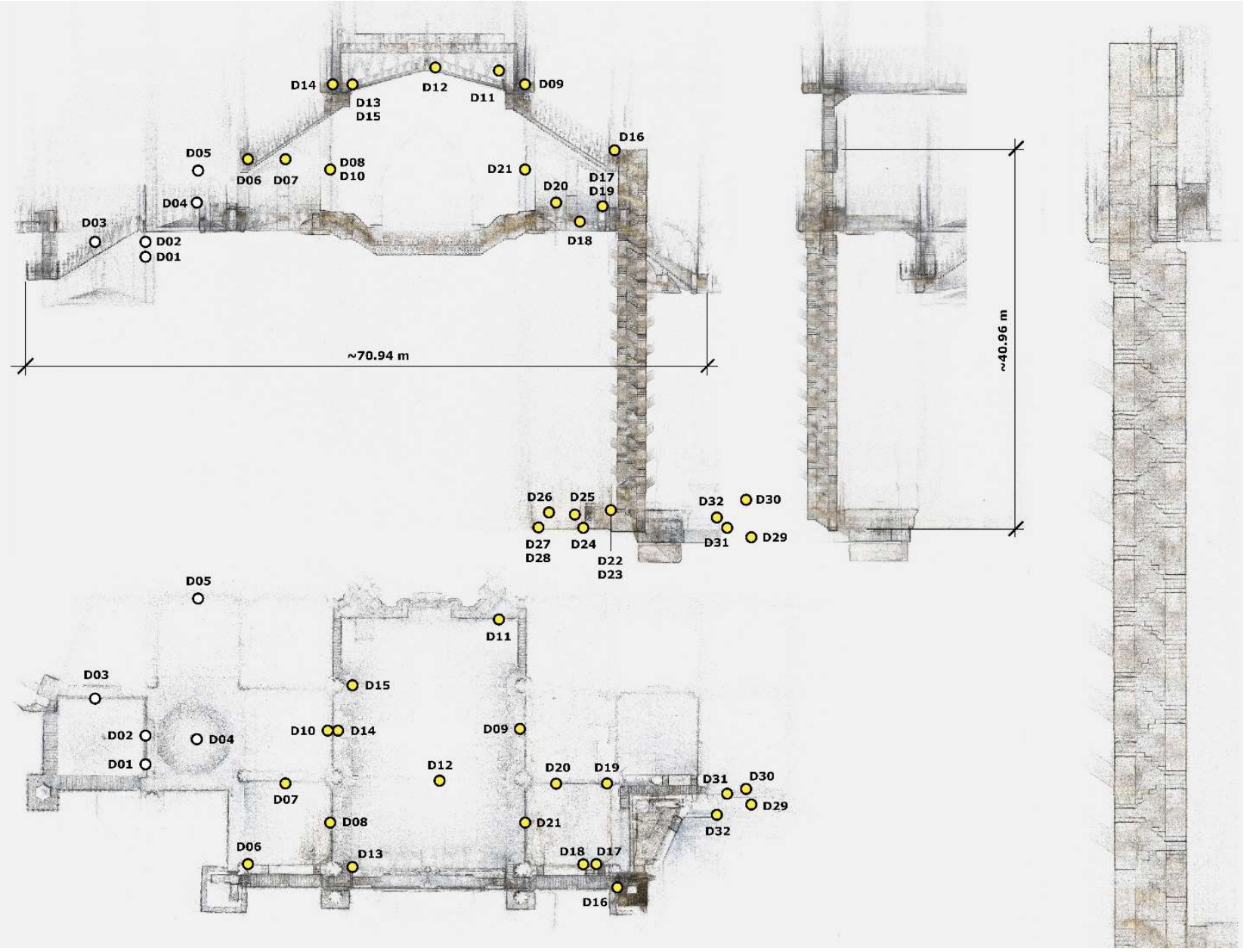

Figure 8. Point cloud result of Path D. Elevation views (top) and top views (bottom). On the right side a zoom of the spiral staircase. According to Table 3 "Path D", points in white are used as GCPs in the "max drift check". 
At this point a set of CPs were identified along the paths. Reference coordinates were obtained from existing point clouds with an accuracy of $\sim 2 \mathrm{~cm}$. Table 2 and Table 3 in this section reports the resulting error (centimetres) for all paths of the two evaluations described in Section 3. In Table 2 we can see the results of Path A, the only closed loop acquisition that resulted in a maximum error on $6.7 \mathrm{~cm}$ for the "global check" and a

\begin{tabular}{|c|c|c|c|c|c|c|c|c|}
\hline \multirow[b]{3}{*}{ ID } & \multicolumn{8}{|c|}{ Path B } \\
\hline & \multicolumn{4}{|c|}{ global check } & \multicolumn{4}{|c|}{ max drift check } \\
\hline & err. & $X$ & $\mathrm{Y}$ & $\mathrm{Z}$ & err. & $\mathrm{X}$ & $\mathrm{Y}$ & $Z$ \\
\hline B01 & 2.6 & 0.9 & 2.3 & 0.9 & 1.6 & 0.1 & 1.6 & -0.5 \\
\hline B02 & 5.5 & 2.6 & 0.3 & 4.8 & 3.8 & 1.9 & -0.3 & 3.3 \\
\hline B03 & 1.9 & 1.5 & 1.2 & 0.2 & 1.3 & 0.5 & 0.3 & -1.1 \\
\hline B04 & 2.1 & 0.8 & 1.9 & 0.6 & 1.2 & 0.0 & 1.1 & -0.6 \\
\hline B05 & 1.8 & 1.4 & 1.1 & -0.1 & 1.6 & 0.6 & 0.3 & -1.4 \\
\hline B06 & 2.1 & 0.8 & 1.9 & 0.3 & 1.7 & 0.2 & 1.4 & -0.9 \\
\hline B07 & 1.2 & -0.1 & 1.1 & 0.4 & 1.5 & -1.2 & 0.0 & -1.0 \\
\hline B08 & 1.1 & 0.9 & -0.6 & 0.1 & 1.6 & 0.2 & -1.2 & -1.0 \\
\hline B09 & 1.5 & -0.2 & 1.3 & 0.7 & 1.1 & -1.1 & 0.0 & -0.2 \\
\hline $\mathrm{B} 10$ & 0.6 & -0.3 & 0.3 & 0.4 & 1.6 & -0.9 & -1.3 & 0.3 \\
\hline B11 & 1.4 & -1.0 & 0.7 & 0.7 & 2.1 & -1.4 & -1.1 & 1.2 \\
\hline B12 & 1.0 & -0.6 & -0.5 & 0.6 & 2.9 & -0.7 & -2.3 & 1.5 \\
\hline B13 & 1.5 & -0.5 & -0.8 & 1.2 & 3.2 & -0.5 & -2.4 & 2.1 \\
\hline B14 & 0.7 & -0.1 & -0.1 & 0.7 & 1.9 & -0.1 & -1.4 & 1.3 \\
\hline B15 & 1.0 & -0.8 & 0.2 & 0.5 & 1.5 & -1.0 & -1.0 & 0.6 \\
\hline B16 & 1.0 & 0.5 & 0.8 & 0.0 & 0.7 & 0.0 & 0.0 & -0.7 \\
\hline B17 & 1.1 & -0.8 & 0.6 & -0.4 & 2.5 & -1.9 & -1.0 & -1.3 \\
\hline B18 & 2.6 & -1.8 & -0.9 & -1.8 & 4.2 & -2.6 & -2.7 & -1.9 \\
\hline B19 & 5.0 & -3.7 & -2.1 & -2.5 & 6.4 & -4.3 & -4.2 & -2.0 \\
\hline B20 & 3.7 & -0.5 & -3.4 & -1.2 & 5.3 & -0.7 & -5.3 & -0.3 \\
\hline B21 & 4.7 & -1.7 & -4.0 & -1.8 & 6.0 & -1.9 & -5.6 & -1.2 \\
\hline B22 & 2.7 & -0.5 & -2.4 & -1.0 & 4.0 & -0.9 & -3.8 & -0.9 \\
\hline B23 & 1.3 & 0.6 & -0.5 & -1.0 & 2.4 & -0.1 & -1.7 & -1.7 \\
\hline B24 & 3.9 & 2.8 & 1.8 & -2.0 & 3.9 & 2.0 & 0.8 & -3.2 \\
\hline $\max$ & 5.5 & 2.6 & 0.3 & 4.8 & 6.4 & -4.3 & -4.2 & -2.0 \\
\hline
\end{tabular}

\begin{tabular}{|c|c|c|c|c|c|c|c|c|}
\hline \multirow[b]{3}{*}{ ID } & \multicolumn{8}{|c|}{ Path C } \\
\hline & \multicolumn{4}{|c|}{ global check } & \multicolumn{4}{|c|}{ max drift check } \\
\hline & err. & $\mathrm{X}$ & $\mathrm{Y}$ & Z & err. & $X$ & $\mathrm{Y}$ & $Z$ \\
\hline $\mathrm{C} 01$ & 10.3 & -3.5 & -8.5 & 4.6 & 2.4 & 1.4 & -1.9 & 0.1 \\
\hline $\mathrm{C} 02$ & 8.8 & -3.2 & -7.3 & 3.7 & 1.9 & 1.1 & -1.4 & -0.5 \\
\hline $\mathrm{C} 03$ & 7.5 & -5.3 & -3.4 & 4.1 & 2.4 & -1.5 & 1.8 & 0.3 \\
\hline $\mathrm{C} 04$ & 6.8 & -4.2 & -4.1 & 3.5 & 1.8 & -1.0 & 1.5 & 0.1 \\
\hline $\mathrm{C} 05$ & 5.9 & -3.7 & -0.7 & -4.5 & 10.1 & -2.0 & 6.9 & -7.1 \\
\hline $\mathrm{C} 06$ & 2.9 & -0.6 & -1.9 & -2.2 & 8.3 & 1.5 & 6.4 & -5.1 \\
\hline $\mathrm{C} 07$ & 4.3 & -3.2 & -0.5 & -2.9 & 10.3 & -1.6 & 8.5 & -5.5 \\
\hline $\mathrm{C} 08$ & 2.4 & -0.2 & -1.0 & -2.2 & 9.7 & 0.7 & 8.6 & -4.4 \\
\hline $\mathrm{C} 09$ & 4.0 & 1.5 & 2.3 & -2.8 & 13.5 & 1.8 & 12.5 & -4.7 \\
\hline $\mathrm{C} 10$ & 3.8 & 2.4 & 2.8 & -1.1 & 13.9 & 2.1 & 13.5 & -2.6 \\
\hline $\mathrm{C} 11$ & 3.7 & 0.9 & 3.4 & -1.3 & 14.8 & -0.1 & 14.6 & -2.5 \\
\hline $\mathrm{C} 12$ & 5.3 & 3.0 & 4.4 & 0.2 & 16.2 & 1.4 & 16.1 & -0.7 \\
\hline $\mathrm{C} 13$ & 5.8 & 2.8 & 4.8 & 1.4 & 17.3 & 0.6 & 17.2 & 1.0 \\
\hline $\mathrm{C} 14$ & 7.1 & 6.2 & 3.5 & -0.3 & 16.9 & 3.5 & 16.5 & -0.4 \\
\hline $\mathrm{C} 15$ & 9.6 & 7.2 & 6.3 & -0.5 & 19.6 & 4.3 & 19.1 & -0.6 \\
\hline $\max$ & 10.3 & -3.5 & -8.5 & 4.6 & 19.6 & 4.3 & 19.1 & -0.6 \\
\hline
\end{tabular}

maximum error of $13.3 \mathrm{~cm}$ for the "max drift check". At points A11-A12 there is already an accumulated error of $\sim 5 \mathrm{~cm}$ and after that the error increases the most, where the path continues in the outdoor environment of the rooftop.

\begin{tabular}{|c|c|c|c|c|c|c|c|c|}
\hline \multirow[b]{3}{*}{ ID } & \multicolumn{8}{|c|}{ Path D } \\
\hline & \multicolumn{4}{|c|}{ global check } & \multicolumn{4}{|c|}{ max drift check } \\
\hline & err. & $X$ & $\mathrm{Y}$ & $\mathrm{Z}$ & err & $X$ & $\mathrm{Y}$ & $\mathrm{Z}$ \\
\hline D01 & 8.3 & -3.8 & 3.9 & -6.3 & 1.1 & -0.1 & -0.8 & -0.8 \\
\hline D02 & 8.1 & -3.1 & 4.8 & -5.7 & 0.6 & 0.6 & 0.0 & -0.1 \\
\hline D03 & 9.6 & -4.2 & 3.4 & -7.9 & 1.7 & -0.4 & -1.5 & -0.6 \\
\hline D04 & 5.9 & -2.9 & 3.4 & -3.9 & 1.6 & 1.5 & -0.5 & 0.3 \\
\hline D05 & 12.9 & -9.4 & 8.6 & -2.2 & 3.5 & -1.6 & 2.9 & 1.2 \\
\hline D06 & 2.9 & -1.2 & -2.5 & -0.9 & 6.7 & 4.7 & -4.7 & 1.2 \\
\hline D07 & 2.1 & -1.9 & 0.7 & -0.4 & 3.9 & 3.2 & -1.9 & 1.1 \\
\hline D08 & 2.7 & -1.7 & 1.7 & -1.4 & 3.6 & 3.2 & -0.8 & -1.4 \\
\hline D09 & 7.5 & 4.0 & 0.7 & 6.3 & 9.8 & 9.7 & 0.2 & 1.1 \\
\hline D10 & 6.2 & -0.8 & 6.2 & -0.1 & 5.1 & 3.8 & 3.4 & 0.3 \\
\hline D11 & 8.0 & 1.3 & 3.7 & 6.9 & 8.1 & 7.0 & 2.7 & 3.2 \\
\hline D12 & 3.9 & 1.9 & -1.2 & 3.2 & 8.6 & 8.4 & -1.6 & 0.4 \\
\hline D13 & 3.5 & -0.1 & -2.3 & 2.7 & 7.5 & 6.7 & -3.0 & 1.8 \\
\hline D14 & 3.2 & -0.8 & 0.5 & 3.0 & 6.6 & 5.6 & -0.7 & 3.4 \\
\hline D15 & 4.0 & -0.6 & 1.0 & 3.8 & 6.8 & 5.6 & -0.4 & 3.8 \\
\hline D16 & 8.8 & 7.1 & -1.6 & 5.0 & 12.1 & 11.4 & -2.4 & -3.5 \\
\hline D17 & 7.5 & 7.0 & -2.0 & 1.7 & 12.5 & 10.1 & -4.0 & -6.1 \\
\hline D18 & 6.8 & 6.5 & -1.8 & 1.4 & 11.8 & 9.3 & -4.1 & -6.0 \\
\hline D19 & 6.7 & 5.4 & 0.9 & 3.8 & 9.1 & 8.2 & -1.4 & -3.8 \\
\hline D20 & 5.0 & 4.4 & -0.2 & 2.3 & 8.8 & 7.5 & -2.6 & -3.9 \\
\hline D21 & 5.5 & 4.0 & -3.0 & 2.2 & 9.9 & 8.0 & -4.8 & -3.3 \\
\hline D22 & 2.7 & 2.5 & -0.9 & 0.5 & 11.5 & -1.5 & -8.6 & -7.4 \\
\hline D23 & 3.8 & -0.9 & -3.7 & 0.7 & 14.3 & -4.9 & -11.4 & -7.2 \\
\hline D24 & 6.4 & 6.3 & 0.1 & -0.9 & 11.6 & 2.3 & -7.7 & -8.3 \\
\hline D25 & 5.7 & 5.5 & -1.5 & -0.5 & 12.1 & 1.7 & -9.2 & -7.7 \\
\hline D26 & 9.6 & 9.0 & -3.1 & 0.4 & 13.6 & 5.5 & -10.8 & -6.0 \\
\hline D27 & 10.0 & 7.2 & -6.8 & -1.6 & 17.0 & 3.3 & -14.9 & -7.5 \\
\hline D28 & 13.2 & 1.6 & -12.9 & -2.2 & 22.8 & -2.6 & -21.4 & -7.6 \\
\hline D29 & 11.6 & -8.6 & 7.8 & -0.4 & 18.4 & -13.9 & 0.0 & -12.1 \\
\hline D30 & 12.5 & -10.1 & 7.3 & -1.0 & 19.4 & -14.7 & 0.0 & -12.6 \\
\hline D31 & 11.2 & -8.7 & 7.0 & -1.2 & 18.4 & -13.6 & -0.6 & -12.4 \\
\hline D32 & 8.6 & -5.4 & 6.7 & -0.6 & 15.2 & -9.9 & -0.7 & -11.5 \\
\hline \multirow[t]{3}{*}{ Max } & 13.2 & 1.6 & -12.9 & -2.2 & 22.8 & -2.6 & -21.4 & -7.6 \\
\hline & \multicolumn{8}{|c|}{ Path E } \\
\hline & \multicolumn{4}{|c|}{ global check } & \multicolumn{4}{|c|}{ max drift check } \\
\hline ID & err. & $X$ & $\mathrm{Y}$ & $\mathrm{Z}$ & err. & $\mathrm{X}$ & $\mathrm{Y}$ & $Z$ \\
\hline E01 & 4.3 & 2.6 & 3.5 & -0.4 & 1.1 & 0.9 & 0.3 & -0.5 \\
\hline E02 & 3.0 & 2.0 & 2.1 & -0.5 & 1.3 & 0.2 & -0.8 & -0.9 \\
\hline E03 & 3.9 & 1.1 & 3.2 & 2.0 & 1.7 & -1.0 & 0.4 & 1.4 \\
\hline E04 & 3.4 & 1.3 & 3.1 & 0.5 & 0.2 & -0.1 & 0.2 & 0.1 \\
\hline E05 & 2.9 & 0.8 & 2.5 & 1.2 & 1.1 & -1.1 & 0.1 & 0.0 \\
\hline E06 & 3.5 & -0.5 & -3.2 & -1.5 & 6.6 & -2.6 & -5.2 & -3.1 \\
\hline E07 & 4.3 & -1.8 & -4.0 & 0.1 & 8.0 & -5.2 & -5.8 & -1.7 \\
\hline E08 & 3.9 & -2.4 & -2.4 & -1.8 & 8.4 & -6.3 & -4.1 & -3.9 \\
\hline E09 & 5.8 & -3.0 & -4.9 & 0.4 & 9.3 & -6.5 & -6.4 & -1.9 \\
\hline $\max$ & 5.8 & -3.0 & -4.9 & 0.4 & 9.3 & -6.5 & -6.4 & -1.9 \\
\hline
\end{tabular}

Table 3. Error check of Path B, C, D and E. For "global check" all points are used as CPs, while for "max drift check" points highlighted in grey are used as GCPs in a similarity transformation. Only for Path D an additional check was performed by using GCPs in the bundle adjustment (Bold IDs), the results are described in the conclusions. All errors are expressed in centimetres. 


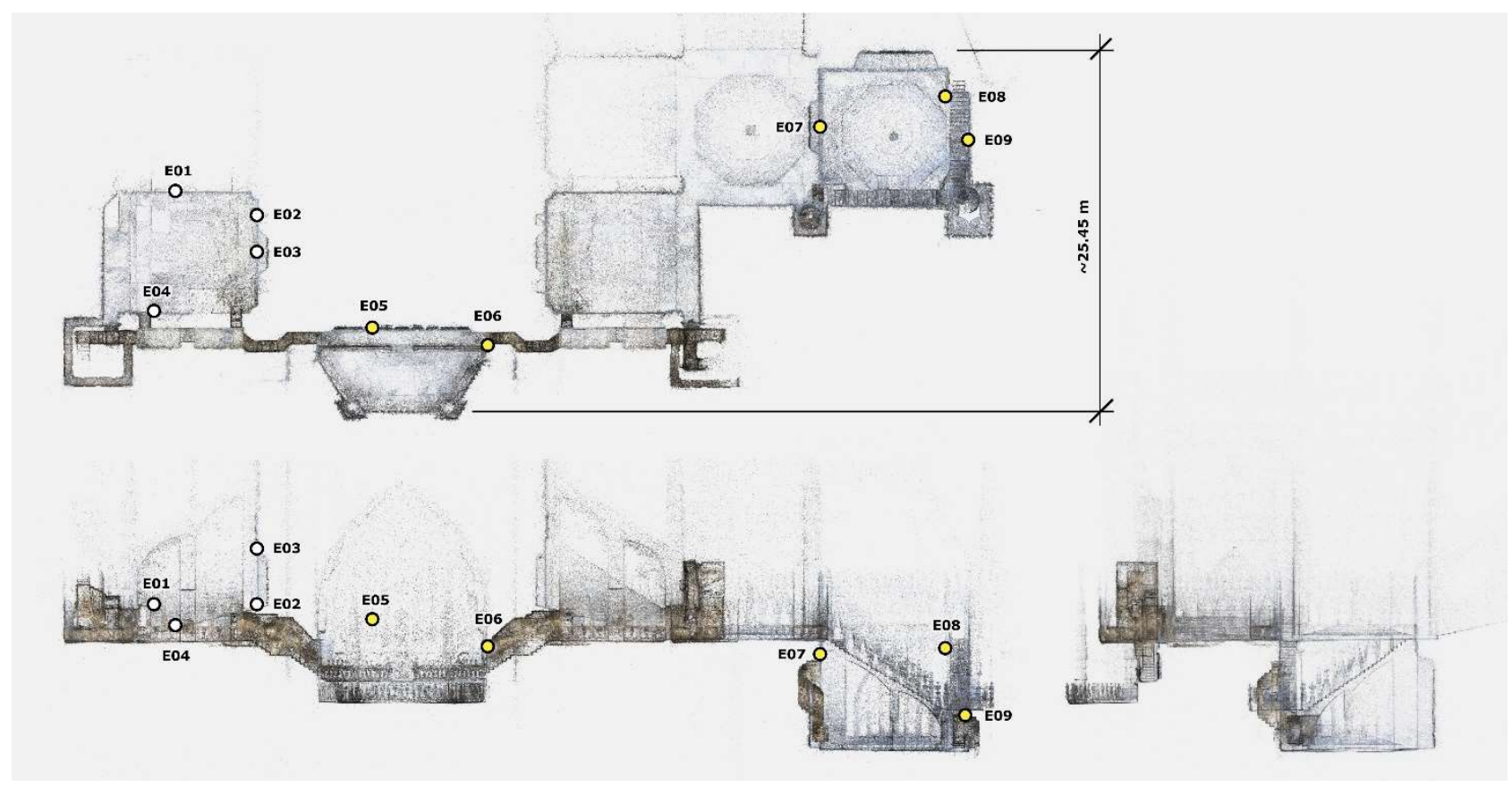

Figure 9. Point cloud result of Path E. Top view (top) and elevation views (bottom): front elevation bottom left and side elevation bottom right. According to Table 3 "Path E", points in white are used as GCPs in the "max drift check".

\begin{tabular}{|c|c|c|c|c|c|c|}
\cline { 2 - 7 } \multicolumn{1}{c|}{} & $\mathrm{N}^{\circ}$ Images & $\mathrm{N}^{\circ}$ Sequences & Acq. duration & Path length & Max error & Drift error (100m) \\
\hline Path A & 13700 & 5 & $70 \mathrm{~min}$ & $\sim 290 \mathrm{~m}$ & $13.3 \mathrm{~cm}$ & $4.6 \mathrm{~cm}$ \\
\hline Path B & 1630 & 4 & $35 \mathrm{~min}$ & $\sim 295 \mathrm{~m}$ & $6.4 \mathrm{~cm}$ & $2.2 \mathrm{~cm}$ \\
\hline Path C & 4460 & 1 & $15 \mathrm{~min}$ & $\sim 245 \mathrm{~m}$ & $19.6 \mathrm{~cm}$ & $8.0 \mathrm{~cm}$ \\
\hline Path D & 12015 & 8 & $120 \mathrm{~min}$ & $\sim 465 \mathrm{~m}$ & $22.8 \mathrm{~cm}$ & $4.9 \mathrm{~cm}$ \\
\hline Path E & 6100 & 4 & $45 \mathrm{~min}$ & $\sim 256 \mathrm{~m}$ & $9.3 \mathrm{~cm}$ & $3.6 \mathrm{~cm}$ \\
\hline
\end{tabular}

Table 4. Main Data of the five path acquisitions. Path length is computed as the distance travelled by the multicamera in the outward acquisition direction only.

Despite the error, at a visual inspection, no distortions are detectable in the Reconstruction. Showing that the multicamera system can maintain the overall shape of the environment and orthogonality of the different elements. This is confirmed also by the results of "Path C" that is instead an open loop acquisition carried out in only the outward direction. In the "max drift check" it can be noticed that immediately after control point $\mathrm{C} 04$, at $\mathrm{C} 05$ there is an accumulated error of $\sim 10 \mathrm{~cm}$, mostly concentrated in the $\mathrm{Z}$ direction, suggesting a distortion in the reconstruction of the enclosed spiral staircase connecting the two levels of the roofs. After that, the error increases of around $9 \mathrm{~cm}$ in the remaining $\sim 80 \mathrm{~m}$ of passage.

A similar drift error can be observed in the results of "Path E" where a total error of around $9 \mathrm{~cm}$ accumulates in a path length of around $256 \mathrm{~m}$ (Table 4).

A better result is obtained from "Path B" where the "max drift check" resulted in a max error of $6.4 \mathrm{~cm}$ that would be just suitable for 1:100 representation scale even without any constraints. Results are also positive for "Path D" where the entire open-ended path, that is the most complex of the set, resulted in a "max drift check" error of $22 \mathrm{~cm}$ that considering the length of the instrument trajectory produced a contained drift deviation (Table 4). Moreover, for "Path D", the visual inspection of the spiral staircase, the narrow connection between the roof level and the grounds, shown no noticeable distortions or bends in the reconstruction. This is confirmed by the errors that did not increase significantly from the top to the bottom of the staircase. Table 4 summarize the main data of the five acquisitions and helps to draw some conclusion on the behaviour of the multicamera system. The "path length" is the distance travelled by the multicamera system in the outward direction. The "drift error" is computed from the max error obtained in the "max drift check" for each path divided then by the path length, results are normalized on a $100 \mathrm{~m}$ metres run. The multicamera system average performance can be described best by Path A, Path D and Path $E$ with an average drift error in the order of $\sim 4.5 \mathrm{~cm}$ every $100 \mathrm{~m}$ of a two-ways trajectory. The drift error is double for Path $C$, where the entire acquisition consists of the outward acquisition, with no return path ever acquired. The drift error is instead half the average for Path B, for this test the acquisition was slower since the vaults rooms acquired are larger than the average passage and the acquisition focused more to acquire all the geometry.

\section{CONCLUSIONS}

The results of the test show the potential of the proposed approach. The multicamera system was always able to reconstruct the whole paths in all their parts and in short time (Table 4). No distortion or bends in the reconstructed trajectory are visible. In the worst-case scenario of unconstrained open loop paths, the overall shape and orthogonality of elements are still preserved. Looking at the staircase zoom in Figure 8 and at the mesh model view of Figure 10 it is possible to conclude that the proposed system can be used effectively to generate $3 \mathrm{D}$ models and to trace $2 \mathrm{D}$ drawings up to scale 1:50, at least locally. 
Moreover, it can complement other survey techniques to measure complex areas.

On the other hand, the results also prove that, at the current development of the multicamera system, GCPs are needed to constraint, control at improve the results within an architectonic accuracy also globally. for instance, for "Path D", the longest acquired: if some GCPs distributed all along the survey trajectory are used (see Table 3 "Path D", target ID in bold), the root mean square error of the CPs lowers to $3.9 \mathrm{~cm}$ and that of the GCPSs is $3.6 \mathrm{~cm}$ (the reference points accuracy is $\sim 2 \mathrm{~cm}$ ).

For an actual proper 3D survey, the proposed solution yields result suitable for the scale 1:100 up to 1:50. And even in the complete absence of constraints the reconstructions are complete and free from obvious bends and distortions; in this scenario we can expect a drift of about $4.5 \mathrm{~cm}$ every $100 \mathrm{~m}$ measured both ways.

Nevertheless, the unconstrained max drift error should be improved. The main issues encountered during the tests were the following: (i) uneven lighting, the cameras dynamic range is not ideal for all conditions, image processing can be tested to reduce the contrast in the scenes; (ii) relative orientation, for now only the baselines were exploited to constrain the multicamera, rotation can be implemented as well; (iii) length of the processing time, the processing time can be shortened by implementing a real-time pre processing of the data during the acquisition.



Figure 10. 3D view of portion of the mesh model produced from the acquisition of Path A (top).

\section{FUTURE WORKS}

Future developments of the multicamera system can concentrate on two main aspects, the first is the improvement of the usability and the effectiveness of the current solution: this include the implementation of the full multicamera constraints and the implementation of image processing strategy to improve the lighting of the scene which is also critical for the texturing of the model and the colouring of the point cloud. The second aspect is the development of new features that could expand the capabilities of the device: first, there is the implementation of real-time processing and secondly there is the integration of additional positioning and mapping sensors along with the photogrammetric multicamera.

\section{AKKNOWLEDGEMENTS}

The authors would like to thank Veneranda Fabbrica del Duomo di Milano, especially the director Eng. Francesco Canali for supporting this work by making all tests possible and Elizaveta Gradusova for the work carried out for her master thesis.

\section{REFERENCES}

Barazzetti, L., Previtali, M., and Roncoroni, F., 2017. 3d modelling with the samsung gear 360. Int. Arch. Photogramm. Remote Sens. Spatial Inf. Sci., Vol. XLII-2/W3, pp. 85-90.

Barazzetti, L., Previtali, M., \& Scaioni, M., 2020. Procedures for condition mapping using $360^{\circ}$ images. ISPRS International Journal of Geo-Information, Vol. 9(1), pp. 34.

Iglewicz, B., \& Hoaglin, D., 1993. Volume 16: How to Detect and Handle Outliers. American Society for Quality Control, Milwaukee, WI. Quality Press.

Koehl, M., Delacourt, T., and Boutry, C., 2016. Image capture with synchronized multiple-cameras for extraction of accurate geometries. Int. Arch. Photogramm. Remote Sens. Spatial Inf. Sci., Vol. XLIB1, pp. 653-660.

Meyer, D. E., Lo, E., Klingspon, J., Netchaev, A., Ellison, C., and Kuester, F., 2020. TunnelCAM- a HDR spherical camera array for structural integrity assessments of dam interiors. Electronic Imaging, Vol. 2020(7), pp. 227-1.

Nocerino, E., Nawaf, M. M., Saccone, M., Ellefi, M. B., Pasquet, J., Royer, J.-P., and Drap, P., 2018. Multi-camera system calibration of a low-cost remotely operated vehicle for underwater cave exploration. Int. Arch. Photogramm. Remote Sens. Spatial Inf. Sci., Vol. XLII-1, pp. 329-337.

Nocerino, E., Menna, F., Farella, E., and Remondino, F., 2019. 3D Virtualization of an underground semi-submerged cave system. Int. Arch. Photogramm. Remote Sens. Spatial Inf. Sci., Vol. XLII-2/W15, pp. 857-864.

Panella, F., Roecklinger, N., Vojnovic, L., Loo, Y., and Boehm, J., 2020. Cost-benefit analysis of rail tunnel inspection for photogrammetry and laser scanning. Int. Arch. Photogramm. Remote Sens. Spatial Inf. Sci., Vol. XLIII-B2-2020, pp. 1137 1144.

Perfetti, L., Polari, C., \& Fassi, F., 2017. Fisheye photogrammetry: tests and methodologies for the survey of narrow spaces. Int. Arch. Photogramm. Remote Sens. Spatial Inf. Sci., Vol. XLII-2/W3, pp. 573-580.

Perfetti, L., Polari, C., \& Fassi, F., 2018. Fisheye Multi-Camera System Calibration for Surveying Narrow and Complex Architectures. Int. Arch. Photogramm. Remote Sens. Spatial Inf. Sci., Vol. XLII-2, pp. 877-883.

Perfetti, L., 2020. Multi-Camera Rig For 3D Reconstruction: Concept, Design and Accuracy Evaluation. in: Luhmann, T., Schumacher, C., Photogrammetrie - Laserscanning - Optische 3D-Messtechnik, Beiträge der Oldenburger 3D-Tage 2020, pp. 124-131.

Shortis, M., Harvey, E., and Abdo, D., 2009. A review of underwater stereo-image measurement for marine biology and ecology applications. Oceanography and Marine Biology, pp. 257-292.

Teo, T., 2015. Video-based point cloud generation using multiple action cameras. Int. Arch. Photogramm. Remote Sens. Spatial Inf. Sci., Vol. XL-4/W5, pp. 55-60. 